\title{
RZ Cassiopeia: an Eclipsing Binary with a Pulsating Component
}

\author{
Alex Golovin ${ }^{1} \dagger$, and Elena P. Pavlenko ${ }^{2}$ \\ ${ }^{1}$ Main Astronomical Observatory of NAS of Ukraine; Zabolotnoho str., \\ 27, Kyiv, 03680, Ukraine
}

Kyiv National Taras Shevchenko University, Volodymyrska Street, 64, Kyiv, 01033, Ukraine; Visiting astronomer of the Crimean Astrophysical Observatory, Nauchny, Crimea, Ukraine email: astronom_2003@mail.ru

${ }^{2}$ Crimean Astrophysical Observatory, p/o Nauchny 19/17, Crimea, 98409, Ukraine

\begin{abstract}
We report time-resolved $V$ - and $R$-band CCD photometry of the eclipsing binary RZ Cas obtained with 38-cm Cassegrain telescope at the Crimean Astrophysical Observatory during July 2004 - October 2005. The obtained lightcurves clearly demonstrates rapid pulsations with a period of about $22 \mathrm{~min}$. A periodogram analysis of these oscillations is also reported. On 12 January, 2005 (JD 2453383) we observed rapid variability with higher amplitude $\left(\sim 0^{\mathrm{m}} .1\right)$, that perhaps may be interpreted as a high-mass-transfer-rate event and inhomogeneity of the accretion stream. Follow-up observations (both photometric and spectroscopic) of RZ Cas are strictly desirable for more detailed study of such events.
\end{abstract}

Keywords. (stars:) binaries: eclipsing; stars: individual (RZ Cas, GSC 04317-01793, HD 17138); stars: mass loss; stars: oscillations; (stars: variables:) delta Scuti; stars: variables: other.

\section{Introduction}

The A3V+K0 IV eclipsing binary RZ Cas is an active semi-detached Algol system showing complex features in its lightcurve. It is well-known for demonstrating rapid pulsations $(\mathrm{P} \sim 22 \mathrm{~min}$ ) superimposed over its orbital eclipsing lightcurve. A brief overview of previous investigations of RZ Cas can be found in Golovin \& Pavlenko (2006).

\section{Observations}

RZ Cas was observed in July 2004 - October 2005 in the $V$ and $R$ bands from the Crimean Astrophysical Observatory (Ukraine) by the authors, using a 38-cm Cassegrain telescope equipped with an SBIG ST-7 CCD camera, cooled by a Peltier system to about $-20^{\circ} \mathrm{C}$. The field-of-view covered a sky region of $12^{\prime} \times 8^{\prime}$ and the pixel size was $0^{\prime \prime} .9 \times 0^{\prime \prime} .9$. The exposure time was 2.5 for the $R$ band and 5 or $10^{\text {s }}$ for the $V$ band. To minimize dead time we used binning by a factor of 2. Data reduction was done using the "Maxim DL" package. Reduction included bias, dark-frame subtraction and flat fielding using twilight sky exposures. Since the field of RZ Cas is not crowded, the technique of aperture photometry was applied to extract the differential magnitudes. The total number of useful frames was 4365. The brightness of RZ Cas was measured with respect to GSC $4317-1578\left(\alpha=02^{\circ} 48^{\prime} 41^{\prime \prime} .58 ; \delta=+69^{\circ} 35^{\prime} 31^{\prime \prime} .3 ; J 2000.0\right)$, while GSC $4317-1437\left(\alpha=02^{\circ} 48^{\prime} 38^{\prime \prime} .29 ; \delta=+69^{\circ} 37^{\prime} 29^{\prime \prime} .9 ; J 2000.0\right)$ served as a check star. Unfortunately, no suitable comparison and check star could be including in the frames

$\dagger$ Present address: Kyiv National Taras Shevchenko University, Volodymyrska Street, 64, Kyiv 01033, Ukraine 


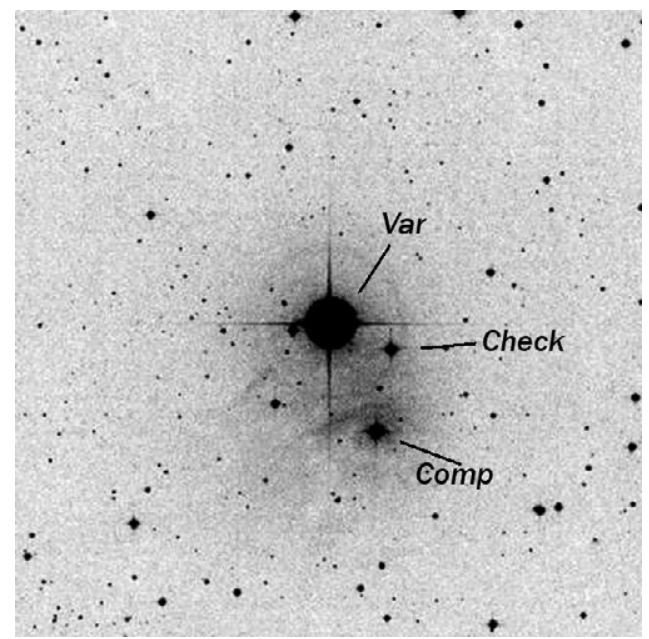

Figure 1. Finding chart.

(stars are at least $4^{\mathrm{m}}$ fainter than the variable star). The photometric error (determined from the difference check star - comparison star) is about $0^{\mathrm{m}} .01$. Figure 1 illustrates a $15^{\prime} \times 15^{\prime}$ image of the RZ Cas region from DSS. Variable, comparison and check stars are marked.
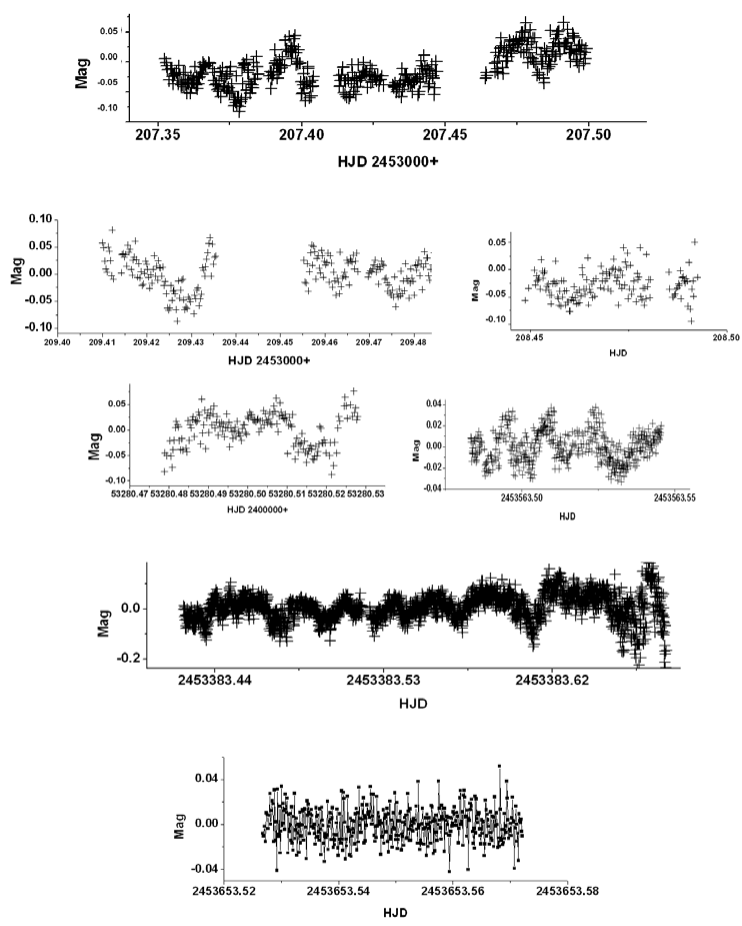

Figure 2. Individual lightcurves. 

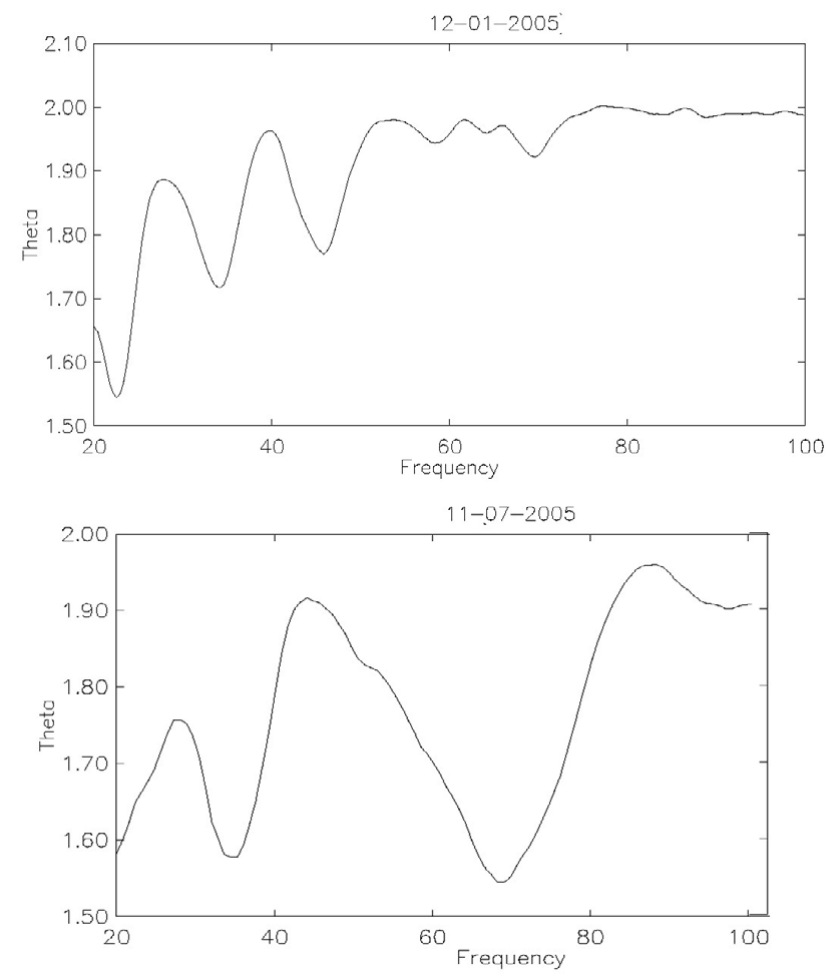

Figure 3. Periodograms.

To rule out the possibility of observing variations caused by the comparison star, independent photometry of comparison star GSC 4317-1578 was performed with respect to check star GSC 4317-1437.

\section{Discussion}

Figure 2 shows brightness variations during the maximal part of the lightcurve. Separate observing runs are manifested. As could be seen, pulsation amplitude could be assumed to be about $0^{\mathrm{m}} .05$ on average. A great deal of interest was caused by the fact that on 12 January, 2005 (JD 2453383) we observed rapid variability with higher amplitude $\left(\sim 0^{\mathrm{m}} \cdot 1\right)$, that perhaps may be interpreted as a high-mass-transfer-rate event and inhomogeneity of the accretion stream. Periodogram analysis (Figure 3) reveals the following periods: For 12, January, 2005 :

$P_{1}=0.041895 \pm 0.000849\left(F_{1}=23.87 \pm 0.48\right)$

$P_{2}=0.021238 \pm 0.000218\left(F_{2}=47.08 \pm 0.48\right)$

$P_{3}=0.017470 \pm 0.000148\left(F_{3}=57.24 \pm 0.48\right)$

$P_{4}=0.014323 \pm 0.000099\left(F_{4}=69.82 \pm 0.48\right)$

For all the other remaining nights: $P_{1}=0.014489 \pm 0.000169\left(F_{1}=69.02 \pm 0.80\right)-$ the period of pulsations of RZ Cas. $P_{2}=0.028354 \pm 0.000646\left(F_{2}=35.27 \pm 0.80\right)-$ almost twice the value of $P_{1}$. 


\section{Conclusions}

We report here photometric observations of RZ Cas' $\delta$ Scuti-like pulsations. We stress attention to the abrupt increase in amplitude (up to $\sim 0^{\mathrm{m}} .1$ ) on 12 January, 2005 that perhaps may be interpreted as a high-mass-transfer-rate event and inhomogeneity of the accretion stream. Follow-up observations (both photometric and spectroscopic) of RZ Cas are strictly desirable for more detailed study of such events.

\section{Acknowledgments}

Alex Golovin is grateful to the Organizing Committee for financial support allowing his participation in the IAU GA. This work was partially supported by the International Workshop for Astronomy e.V., enabling Alex Golovin to do part of this project at Slovakia during 2005 and in the Czech Republic during 2006. It's a great pleasure for Alex Golovin to express here personal thankfulness to Jevgeniy Kachalin for his help with preparation of this manuscript and for the proofreading. We wish to express thankfulness to D. Mkrtichian for valuable discussions.

\section{References}

Golovin, A. \& Pavlenko, E. 2006, JAAVSO, vol. 34, in press

Stellingwerf, R.F. 1978, ApJ, 224, 953 